\title{
Spatially Combined Wideband Interleaved Transmitter
}

\author{
Prasidh Ramabadran, Pavel Afanasyev, Sara Hesami, \\ Darragh McCarthy, Ronan Farrell, John Dooley \\ Dep of Electronic Engineering \\ National University of Ireland, Maynooth \\ Maynooth, Ireland \\ prasidh.ramabadran@mu.ie
}

\author{
Bill O’Brien \\ Nonlinear Systems Limited \\ Dublin, Ireland \\ Bill.obrien@ieee.org
}

\begin{abstract}
In this paper, we propose a novel wideband wireless transmission scheme with spatially combined frequency interleaved transmitters of narrower bandwidths. The proposed technique enables development of seamless scalable bandwidth transmitters for next generation wireless and satellite communications with potential to reuse legacy hardware. The scheme is experimentally validated at Ka Band with instantaneous modulation bandwidths up to $400 \mathrm{MHz}$ achieving transmission bit rates up to $2.039 \mathrm{Gbps}$.
\end{abstract}

Keywords-Physical Layer, Software Defined Radio; Ka Band, Signal Processing;

\section{INTRODUCTION}

Next generation wireless communication systems such as $5 \mathrm{G}$ and High Throughput Satellite (NG-HTS) would feature bandwidths higher than $50 \mathrm{MHz}$ per carrier ranging up to a $\mathrm{GHz}$ or above $[1,2]$. The cost and complexity of implementation of the radio transmission system increases proportional to the wide bandwidths.A block diagram of a typical vector RF modulated heterodyne transmitter is shown in Fig. 1.

A digital signal processor (DSP) generates the vector modulation symbols called basebands (BB) in accordance with the digital data meant for transmission. These digital basebands are applied to a pair of Digital to Analogue Converters (DACs) to generate the equivalent in-phase (I) and quadrature (Q) analogue baseband waveforms. These waveforms are vector upconverted to the required intermediate frequency (IF) and further up-converted to the RF carrier frequency which is amplified by the RF power amplifier (PA) and transmitted. The baseband may be directly up-converted to RF in homodyne transmitters. The maximum operating bandwidth of a transmitter is constrained by the bandwidths of the individual blocks that constitute the transmitter such as the DACs, PA and passive microwave modules. The problem of generating the wideband waveforms has been addressed in the past by interleaving DACs in time and frequency domains. Each interleaving method has its problems such as interleaving spurs, timing offsets and image frequency components to mitigate which have been addressed in [3, 4 and 9] respectively. From a wireless transmission system perspective, the problem in the transmission of wide bandwidths is not limited to DACs alone. In [5], the authors propose a scheme to generate a wideband modulated RF carrier with bandwidth interleaved modulators whose outputs are combined before the PA after appropriate calibration of the paths in the

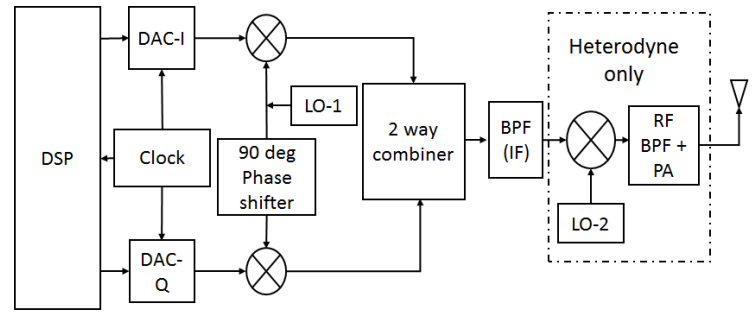

Fig. 1 General Block Diagram of a Wireless Transmitter

modulator. The RF up-converters and PAs are generally integrated with the outdoor units (ODUs) in satellite communication systems where it is not practically viable to implement the scheme described in [5]. PAs are inefficient if operated in the linear region of transfer characteristics. Modern modulation schemes feature modulation of both amplitude (AM) and phase (PM) thereby resulting in a complex modulation envelope. The non-linear characteristics of the PA affect the integrity of the envelope by introducing AM-AM and AM-PM distortions and also result in RF emissions outside the assigned bandwidth which would violate regulatory norms and also intrude the spectrum assigned to other users. PAs also exhibit memory effects where the output signal value at an instant depends on past and present inputs. Memory effects increase with the bandwidth of operation owing to the hysteresis of the components involved in the bias circuits, filters and charge trapping in the active devices. Digital Pre-Distortion (DPD) is applied in transmitters to mitigate the impact of non-linearities. A feedback receiver is required to accomplish DPD in a transmitter which conventionally requires five times the channel bandwidth to acquire the out of band (OOB) emissions. DPD is popularly accomplished by modelling the transmitter as a nonlinear system with memory using basis functions in the form of matrices [8]. The ranks of the matrices increase in proportion to the bandwidth owing to an increase in long term memory effects. To summarize, the overall cost and complexity of wireless communication systems increase proportionally with bandwidth.

In this paper, we present a scheme to extend the concept proposed in [5 and 9] to interleaving transmitters as a whole in the frequency domain and seamlessly combining their outputs spatially at the receiver to cater to wideband transmission 


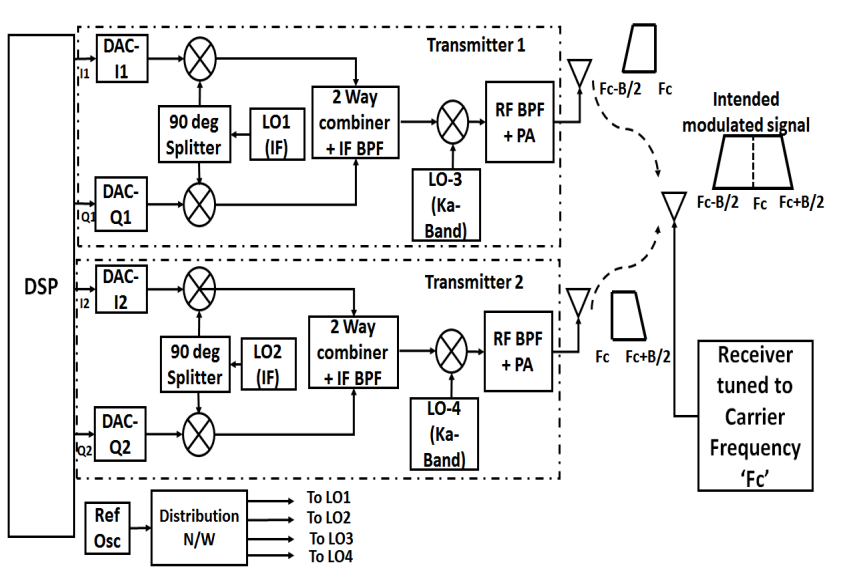

Fig. 2 Bandwidth Interleaved Transmission Scheme

requirements. The remainder of this paper is arranged as follows:

In section II we describe the proposed wideband signal transmission scheme with spatially combined interleaved transmitters and associated signal processing for a nearly seamless reception. The experimentally measured results to validate the technique are presented in section III. Finally, in section IV we cover the main conclusions from this work.

\section{THE PROPOSED SYSTEM DESIGN}

The proposed scheme of generating and transmitting a wideband vector modulated signal with bandwidth interleaved transmitters employing hardware blocks of lower bandwidths is shown in Fig. 2. A wideband modulated signal at carrier frequency $\omega_{c}$ with complex envelope $\mathrm{m}(\mathrm{t})$ may be described mathematically in the form shown in (1).

$$
c(t)=m(t) e^{j \omega c t}
$$

Envelope $\mathrm{m}(\mathrm{t})$ may be represented by a series of complex sinusoids each having an amplitude $a_{n}$ and phase $\Theta_{n}$ that constitute the signal in the form of Discrete Fourier Transform (DFT) having a total of $\mathrm{N}$ frequency bins in the overall bandwidth as shown (2). The instantaneous time $\mathrm{t}=\mathrm{nT}_{\mathrm{s}}$ for $\mathrm{a}$

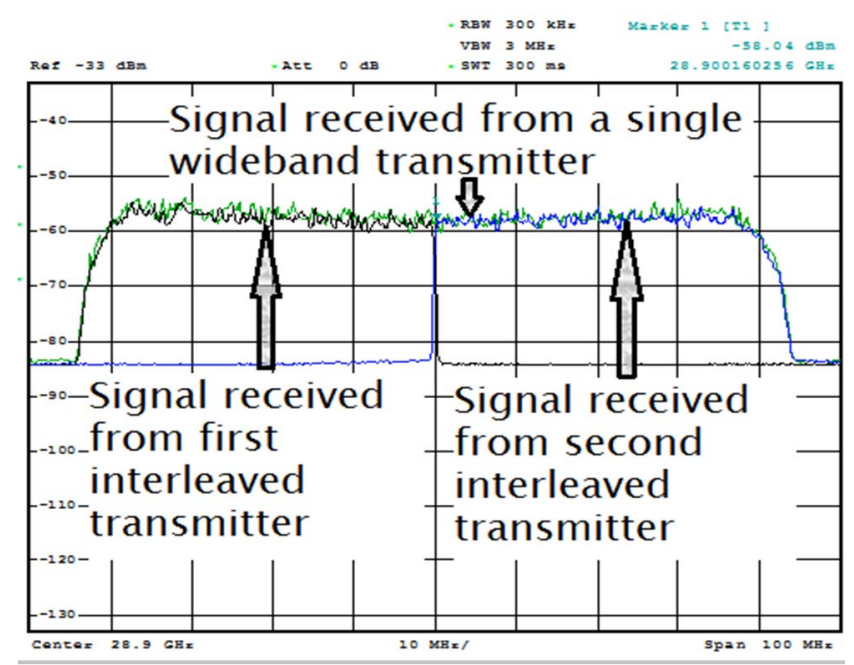

Fig. 3 Received Spectrum of Bandwidth interleaved transmission sampled data system where $\mathrm{n}$ is the sample number and $\mathrm{T}_{\mathrm{s}}$ is the sampling interval.

$$
m\left(n T_{s}\right)=\sum_{n=-\frac{N}{2}}^{n=\frac{N}{2}-1}\left(a_{n} \angle \theta_{n}\right) e^{j \omega n / T s}
$$

This signal is vector up-converted in the modulator to carrier frequency $\omega_{c}=2 \pi f_{c}$. If the overall bandwidth assigned is $\mathrm{B} \mathrm{Hz}$, components at frequency bins $-\mathrm{N} / 2$ to -1 occupy a band $\mathrm{f}_{\mathrm{c}}-\mathrm{B} / 2$ to fc and the components at frequency bins 0 to $N / 2$ occupy the band $\mathrm{fc}$ to $\mathrm{f}_{\mathrm{c}}+\mathrm{B} / 2$ in the transmitted spectrum. Each component in (2) is characterized by a distinct amplitude, phase and frequency. It may therefore be inferred that the transmission of a wide bandwidth signal can be accomplished in parts using more than one transmitter of smaller bandwidths as long as the integrity of the parameters of each frequency component in (2) is preserved at the receiver. This can be accomplished using two spatially combined bandwidth interleaved transmitters employing the architecture shown in Fig. 2 such that the first transmitter transmits the lower half bandwidth components $\left(\mathrm{f}_{\mathrm{c}}\right.$ $\mathrm{B} / 2$ to $\mathrm{f}_{\mathrm{c}}$ ) and the second transmitter transmits the higher half bandwidth components $\left(f_{c}\right.$ to $\left.f_{c}+B / 2\right)$. The two parts are combined spatially in a seamless manner at the receiver as depicted in Fig. 3 to yield the intended wideband modulated signal centred at $\mathrm{f}_{\mathrm{c}}$.

One of the ways to implement the proposed scheme with two heterodyne transmitters shown in Fig. 2 at Ka Band $(28.9 \mathrm{GHz})$ is summarized below. The scheme is also applicable to homodyne architectures and any carrier frequency.

i) Re-sample the complex wideband modulated baseband of interest to Nyquist rate $\left(\mathrm{F}_{\mathrm{s}}\right)$ or above in the digital domain and perform vector upconversion to digital intermediate frequency (IF) centred at ' $\mathrm{Fs} / 4$ ' using cosine component of the digital local oscillator (LO) for the in-phase component and sine component of the LO for the quadrature component of the baseband sampled. Let this be designated as 'IF_cos'. Perform another up-conversion interchanging the sine and cosine LOs to obtain the Hilbert Transform of 'IF cos' and designate this signal as 'IF_sin'.

ii) Compute the Fast Fourier Transform (FFT) of the 'IF cos', extract the components from frequency bins 1 to $F_{s} / 4$ and $3 F_{s} / 4+1$ to $F_{s}$ and invert its spectrum. Compute the Inverse FFT (IFFT) and designate as IF LI. Extract components from FFT bins $\mathrm{F}_{\mathrm{s}} / 4+1$ to $\overline{3 \mathrm{~F}_{\mathrm{s}}} / 4$ and do not invert the spectrum. Compute its IFFT and designate as IF HI.

iii) Compute the Fast Fourier Transform (FFT) of the 'IF_sin', extract the components from frequency bins 1 to $F_{s} / 4$ and $3 F_{s} / 4+1$ to $F_{s}$ and invert its spectrum. Compute the Inverse FFT (IFFT) and 
designate as IF_LQ. Extract components from FFT bins $\mathrm{F}_{\mathrm{s}} / 4+1$ to $\overline{3} \mathrm{~F}_{\mathrm{s}} / 4$ and do not invert the spectrum. Compute its IFFT and designate as IF_HQ.

iv)

Apply IF_LI and IF_LQ to DAC_I1 and DAC_Q1 respectively of the scheme shown in Fig. 2 Apply IF HI and IF HQ to DAC I2 and DAC Q2 respectively of the scheme shown in Fig. 2. Set the frequencies of $\mathrm{LO} 1$ and $\mathrm{LO} 2$ to a common intermediate frequency e.g., $800 \mathrm{MHz}$. If the spectra of IF_LI and IF_LQ were not inverted, the frequency of $\overline{\mathrm{LO}} 1$ would need to be set to frequency IF-BW/2. This is not a problem if the phase mismatches between the two transmitters can be calibrated [6]. This option would be suitable if more than two transmitters are interleaved. LO3 and LO4 are set at the same frequency and selected according to the final carrier frequency of interest. Set them at $28.1 \mathrm{GHz}$ to transmit at carrier frequency $28.9 \mathrm{GHz}$.

v) Set the boresights of the two transmitters in the direction of the intended receiver. Antennas with wide beam-widths (e.g., 120deg sectoral beams) could also be used depending on the end application.

The PAs in each transmitter are needed to transmit only half the total RF power and half the total bandwidth. A common feedback receiver with half the total bandwidth requirement can be switched between the two transmitters to accomplish DPD. This task is not in the scope of the paper and is being pursued as an extension. It is a pre-requisite to calibrate and pre-compensate the RF chains in each transmitter for quadrature offsets and bandwidth dependent impairments [6]. The integrity of the signal at the receiver depends upon the ability to maintain the integrity of amplitudes, phases and timing of the parts of the signal transmitted from the interleaved transmitters. This requires uniformity in gain and phase responses experienced by each part of the signal which could be accomplished by path calibration. It is necessary to calibrate the RF propagation paths between the transmitters and the receiver for gain and phase imbalances.

$$
\begin{aligned}
& \mathrm{y}(\mathrm{n})=\sin \left\{2 \pi\left(\frac{\mathrm{knt}_{\mathrm{s}}}{2}+\mathrm{f}_{\min }\right) \mathrm{nT}_{\mathrm{s}}\right\} \\
& \mathrm{k}=\frac{\mathrm{f}_{\text {max }}-\mathrm{f}_{\text {min }}}{\mathrm{M}}
\end{aligned}
$$

A test signal with a bandwidth equal to that of the intended wideband signal such as a frequency modulated Chirp signal defined by (3) and (4) may be used, where $y(n)$ is the value of the $\mathrm{n}^{\text {th }}$ sample, $\mathrm{T}_{\mathrm{s}}$ is the sampling interval, $\mathrm{M}$ is the total number of samples, $\mathrm{k}$ is the frequency variation parameter, $\mathrm{f}_{\max }$ and $\mathrm{f}_{\text {min }}$ are the maximum and the minimum frequencies respectively.

vi)

This signal is subject to bandwidth interleaved transmission as explained in steps ' $i$ ' to 'iv' and received at the receiver. The FFT of the received signal is computed and the amplitudes and phases of the components received from each transmitter are compared against an ideal Chirp signal. The
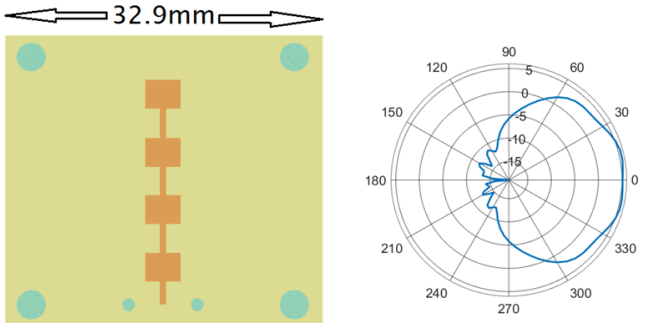

Fig. 4 Gerber screenshot of the antenna designed and its simulated gain pattern $(\mathrm{dBi})$ in azimuth plane.

first numerical differences (derivative or slope) of amplitude and phase spectra are computed. The height of the step at the center frequency in the amplitude and phase would indicate the gain and phase offsets between the two transmit paths respectively. The FFT values also help to train the coefficients of channel equalizer filter in the receiver as in a general case with pilot signals.

vii) The offsets in transmission path 2 relative to 1 thus computed may be either conveyed to Tx2 over a narrow band reverse link for offset tuning. This will enable the seamless reception of the two half bandwidth signals at the receiver which when combined would resemble the intended wideband modulated signal. A narrow band beacon signal on the reverse link will also serve to identify the relative offsets in the two transmit paths when captured by the two transmitting antennas and evaluated owing to channel reciprocity.

A predetermined Orthogonal Frequency Division Multiplexing (OFDM) signal with sub-carriers modulated at lowest order could also be used instead of Chirp for path calibration. Each of these is validated in section III.

\section{EXPERIMENTAL VALIDATION}

The experimental validation was carried out for a single carrier 8PSK DVB-S2 and a 64 QAM-OFDM signal with an intent of proving a multi-standard radio case. The transmitter was built as per the block diagram shown in Fig. 2 for transmission at carrier frequency $28.9 \mathrm{GHz}$. The IF was selected at $800 \mathrm{MHz}$ which was then up-converted to $28.9 \mathrm{GHz}$. LO3 and LO4 were set at $14.05 \mathrm{GHz}$ and were multiplied in frequency by a factor of 2 owing to better phase noise performance than using an oscillator directly at $28.1 \mathrm{GHz}$ to mix with the IF at $800 \mathrm{MHz}$ to yield the modulated half bandwidth carriers at $28.9 \mathrm{GHz}$ at power $+8 \mathrm{dBm}$ and transmitted through four element linear array patch antennas. The receiver consisted of a similar

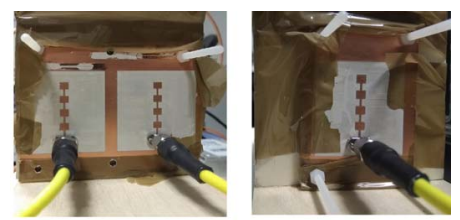

Fig. 5 Transmitting (left) and receiving (right) antennas 
antenna and an RF front end which down-converted the received signal to an IF at $600 \mathrm{MHz}$. This was followed by an Analog to Digital Converter (ADC) clocked at 3Gsps that digitized the IF. Further down-conversion and demodulation were accomplished in the digital domain. The block diagram of the receiver is not shown due to space constraints. The antennas were designed to have beam-widths of 120 degrees in the azimuth plane as shown in Fig. 4 and etched on RO3003 substrate from Rogers Corporation. The two transmitting antennas were fabricated on the same piece of substrate at a spacing of eight wavelengths with a ground plane in between. A wooden test jig was designed with a propagation path length of a meter between the transmitters and the receiver. An overall path loss of $51 \mathrm{~dB}$ was observed between the transmitter and the receiver including the losses in the connecting cables. The evaluation was performed in a regular laboratory environment instead of an anechoic chamber to emulate a real use case scenario that would include multipath propagation effects.

As the first case, a DVB-S2 8PSK signal at symbol rate 80MSPS with Root Raised Cosine (RRC) filtering of rolloff factor $\alpha=0.1$, oversampling ratio (OSR) of 4 was chosen. The effective bandwidth and sample rate post filtering were 88 $\mathrm{MHz}$ and $320 \mathrm{Msps}$ respectively. The signal was resampled to 200Msps and subject to frequency domain partitioning, calibration and interleaved transmission as described in section II with each DAC set at sample rates $100 \mathrm{Msps}$. A vector signal analyzer (VSA) tuned at $28.9 \mathrm{GHz}$ was used as the receiver. This case was chosen since $8 \mathrm{PSK}$ is a single carrier modulation scheme and is more susceptible to offsets and imbalances between the two interleaved paths whose impact would be observed when demodulated by a standard instrument like a VSA. The recorded spectrum is as shown in Fig. 3. This was found comparable to the spectrum of the signal received from a single wideband transmitter connected to the first transmitting antenna. The screenshots of constellation demodulated by the VSA in both cases without channel equalizer is shown in Fig. 6 . The error vector magnitude (EVM) measured by the VSA at a signal to noise ratio (SNR) of $22 \mathrm{~dB}$ were $6.2 \%$ and $6.4 \%$ for the interleaved and single transmitter cases respectively.

The second case chosen was also 8PSK but at symbol rate of $400 \mathrm{Msps}$ and bandwidth of $440 \mathrm{MHz}$ to support transmission over next generation high throughput satellites (NG-HTS) expected to have bandwidths up to $500 \mathrm{MHz}$ per beam $[2,7]$. The signal was partitioned in the frequency domain and transmitted through bandwidth interleaved transmitters each having half the required bandwidth and sample rate. The received signal was down-converted to $600 \mathrm{MHz}$, digitized and demodulated in digital domain on MATLAB as mentioned in the beginning of the section. The inclusion of a low noise RF front end improved the SNR by $11 \mathrm{~dB}$ compared to that observed in the VSA. A channel equalizer filter was trained using the received parameters of a chirp signal of the same bandwidth which was subject to similar interleaved transmission. The mean EVM calculated over 32768 samples without equalization was $12 \%$ but resulted in no bit errors and hence the equalizer trained was not applied. The spectrum of the signal received at the receiver's antenna and the demodulated constellation are shown in Fig. 7 and Fig. 8 respectively.
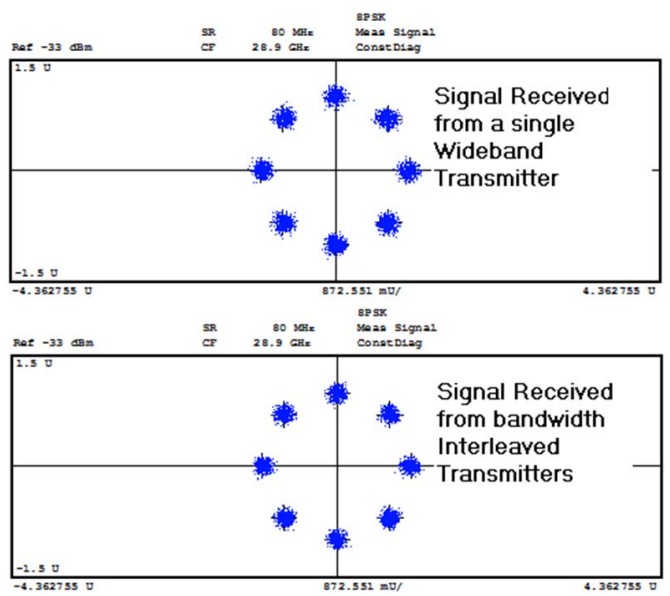

Fig. 6 Comparison of constellations demodulated by VSA at $28.9 \mathrm{GHz}$.

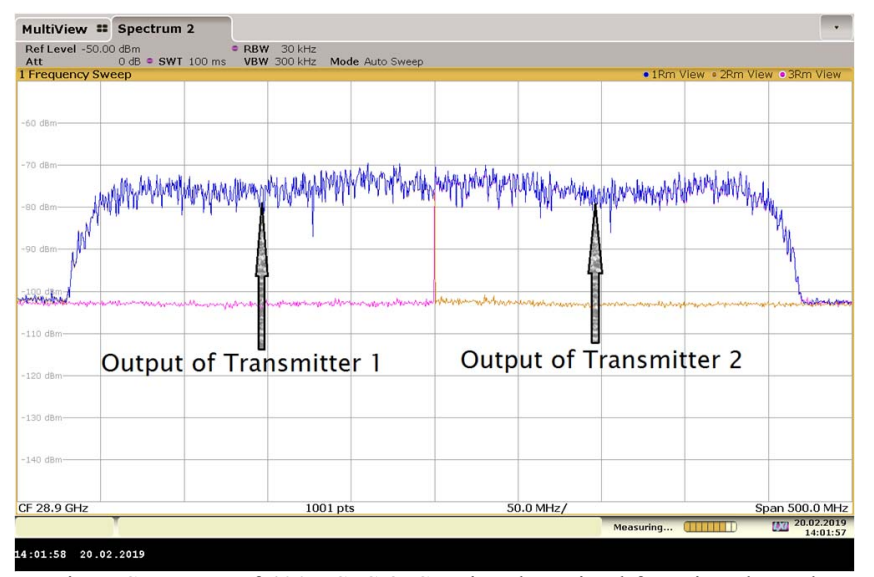

Fig. 7 Spectrum of 400MSPS 8PSK signal received from interleaved transmitters centered at $28.9 \mathrm{GHz}$

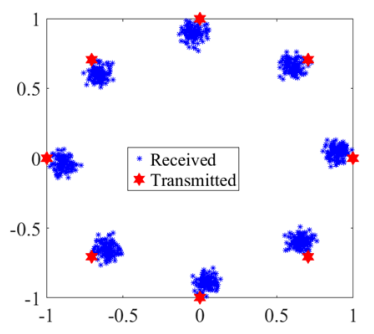

Fig. 8 Demodulated 8PSK constellation at 400MSPS

The third case selected was a 2048 point OFDM signal with a subcarrier spacing of $240 \mathrm{kHz}$ and with 1480 occupied subcarriers including 64 pilot sub-carriers. The sub-carrier spacing was chosen in accordance with 5G-NR specifications at a sample rate of $491.52 \mathrm{MHz}$ with an aim of achieving a throughput of 2.039Gbps using 1416 sub-carriers for data over an effective bandwidth of $355.2 \mathrm{MHz}$. This signal was transmitted with two spatially combined bandwidth interleaved transmitters with each one transmitting half of the bandwidth as described in section II. A BPSK modulated OFDM signal with 
the same number of sub-carriers was first transmitted in the proposed interleaved manner to probe the channel and design an equalizer filter at the receiver. This was followed by transmission of the intended QAM 64 modulated OFDM signal with the interleaved transmitters after the required signal processing described in section II which was received, subject to equalization and demodulation. The length of the cyclic prefix sat set at 128 samples. The calculated mean EVM after equalization was $3.12 \%$ over 16992 symbols (12 OFDM frames). The spectrum of the signal received at the receiver's antenna and the demodulated constellation are as shown in Fig. 9 and Fig. 10 respectively. As each subcarrier represents 6 bits due to QAM64 modulation, a total of 1416 data sub-carriers occupying a bandwidth of $339.84 \mathrm{MHz}(240 \mathrm{kHz}$ X 1416) yield an effective transmission bit rate of $2.039 \mathrm{Gbps}$.

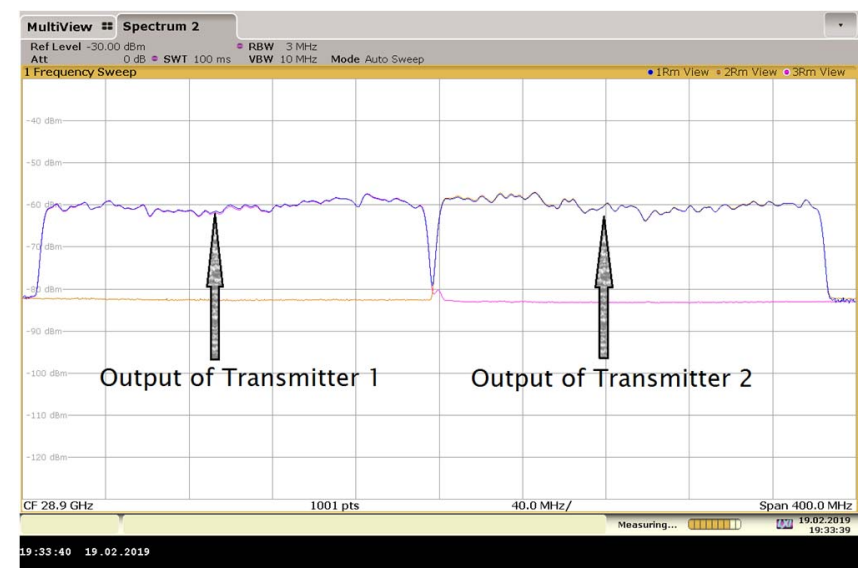

Fig. 10 Spectrum of $355.2 \mathrm{MHz}$ wide QAM64 modulated OFDM signal received from interleaved transmitters centered at $28.9 \mathrm{GHz}$

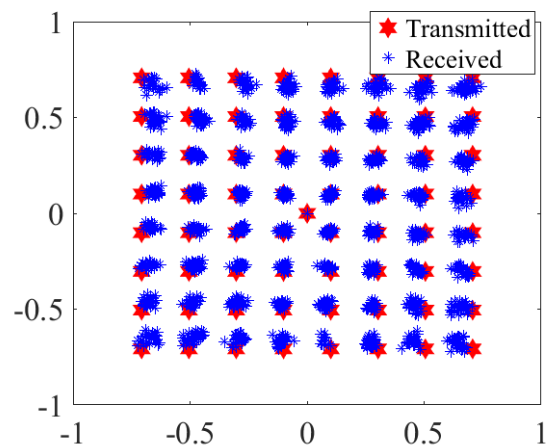

Fig.9 Demodulated constellation of QAM64 OFDM signal of bandwidth 355.2MHz transmitted through interleaved transmitter.

Future Work: Include high power RF power amplifiers in the transmit chains with DPD and also extend the principle to bandwidth interleaved reception.

\section{CONCLUSION}

The proposed scheme for wideband signal generation and transmission using spatial recombination of lower bandwidth transmitters has been demonstrated. Comparable performance is achieved to that of direct wide bandwidth signal modulation and transmission. It has also been demonstrated that the proposed scheme enables the use of same hardware in part or whole for both satellite and $5 \mathrm{G}$ terrestrial communications in $\mathrm{Ka}$ Band. Inherent from its architecture, the proposed scheme is scalable and adaptable to increased transmission bandwidths as it requires only the inclusion of additional interleaved stages. This enables the reuse of existing low bandwidth legacy transmitter hardware either in part or whole for next generation wireless transmission with wider bandwidths.

\section{ACKNOWLEDGMENT}

This publication has emanated from research conducted with the financial support of Science Foundation Ireland (SFI) and is co-funded under the European Regional Development Fund under Grant Number 13/RC/2077. We are grateful to Rogers Corporation for the free samples of low loss RF substrate RO3003 which were used to make the antennas used in this work. We also acknowledge the support provided by Mr. James Kinsella, NUI Maynooth in this project.

\section{REFERENCES}

[1] European Conference Of Postal and Telecommunications Administrations, 'Draft CEPT Report 67 PF_1'.

[2] O. Vidal, G. Verelst, J. Lacan, E. Alberty, J. Radzik and M. Bousquet, "Next generation High Throughput Satellite system," 2012 IEEE First AESS European Conference on Satellite Telecommunications (ESTEL), Rome, 2012, pp. 1-7.

[3] C. Schmidt, C. Kottke, V. Jungnickel and R. Freund, "Enhancing the Bandwidth of DACs by Analog Bandwidth Interleaving," Broadband Coverage in Germany; 10. ITG-Symposium, Berlin, Germany, 2016, pp. $1-8$.

[4] E. Olieman, A. Annema and B. Nauta, "An Interleaved Full Nyquist High-Speed DAC Technique," in IEEE Journal of SolidState Circuits, vol. 50, no. 3, pp. 704-713, March 2015. doi: 10.1109/JSSC.2014.2387946

[5] P. Ramabadran, S. Madhuwantha, P. Afanasyev, R. Farrell and J. Dooley, "Wideband Interleaved Vector Modulators for 5G Wireless Communications," 2018 IEEE MTT-S International Microwave Workshop Series on 5G Hardware and System Technologies (IMWS-5G), Dublin, Ireland, 2018, pp. 1-3. doi: 10.1109/IMWS-5G.2018.8484496

[6] P. Ramabadran, S. Madhuwantha, P. Afanasyev, R. Farrell, L. Marco, S. Pires and J. Dooley, "Digitally Assisted Wideband Compensation of Parallel RF Signal Paths in a Transmitter" 91st ARFTG conference, Philadelphia, USA, 2018 DOI: 10.1109/ARFTG.2018.8423839

[7] APSCC-Q-3-2013 Newsletter-David Rehbehn.

[8] D. R. Morgan, Z. Ma, J. Kim, M. G. Zierdt and J. Pastalan, "A Generalized Memory Polynomial Model for Digital Predistortion of RF Power Amplifiers," in IEEE Transactions on Signal Processing, vol. 54, no. 10, pp. 3852-3860, Oct. 2006. doi: 10.1109/TSP.2006.879264

[9] T. Jyo, H. Yamazaki, M. Nagatani, H. Hamada, H. Fukuyama and H. Nosaka, "An 80-Gbaud Transmitter using Bandwidth Interleaving with Sideband Cancelling Method," 2018 48th European Microwave Conference (EuMC), Madrid, 2018, pp. 247-250. doi: 10.23919/EuMC.2018.8541788 